\title{
A SIMPLE GAUSSIAN APPROXIMATION FOR THE ONE-EODY DENSITY MATRIX
}

\section{J. MEYER}

Institut de Physique Nucléaire de Lyon (et IN2P3), Université de Lyon-I, 43 Bd. du II Novembre 1918, 69622 Villeurbanne Cedex, France ${ }^{I}$ and GANIL, BP 5027, 14021 Caen Cedex, France

\section{J. BARTEL}

Institut für Theoretische Physik, Universität Regensburg, 8400 Regensburg, Fed. Ref. Germany and Institut de Physique Nucléaire de Lyon (et IN2P3), Université de Lyon-I, 43 Bd. du 11 Novembre 1918, 69622 Villeurbanne Cedex, France

\section{BRACK}

Institut für Theoretische Physik, Universität Regensburg, 8400 Regensburg, Fed. Rep. Germany ${ }^{\prime}$ and Laboratoire de Physique Théorique, Université de Bordeaux I, 33170 Gradignan. France

\section{P. QUENTIN}

Laboratoire de Physique Théorique, Université de Bordeaux 1, 33170 Gradignan, Frınce ' and GANIL, BP 5027, 14021 Caen Cedex, France

and

\section{S. AICHER}

Institut für Theoretische Physik, Universität Regensburg, 8400 Regensburg, Fed. Ref. Germany

Received 28 November 1985; revised manuscript received 7 March 1986

\begin{abstract}
A simple form of the nonlocal one-body density matrix $\rho\left(\boldsymbol{r}, \boldsymbol{r}^{\prime}\right)$ is proposed whose parameters are determined by imposing the correct local semiclassical kinetic energy density and the projector character of $\rho$ in an integrated form. The validity of this approach is assessed for physical quantities sensitive to the nonlocal nature of the density matrix such as the momentum distribution in the nucleus, the exchange energies of the Gogny and the Coulonb force and the two-body center-of-mass correction for various nuclei.
\end{abstract}

In static Hartree-Fock (HF) calculations using Skyrme effective forces, one needs to know the nonlocal one-body density matrix $\rho\left(r, r^{\prime}\right)$ only through the local densities $\tau(r), J(r)[1]$, and some other local functions in cases where $\rho$ does not have even timereversal symmetry [2]. However, there are a lot of situations where a full knowledge of $\rho\left(r, r^{\prime}\right)$ or equi-

\footnotetext{
1 Permanent address.
}

valently of its Wigner transform $f(R, p)$ is required. This is obviously the case whenever one has to compute expectatiorı values for Slater-determinant wavefunctions of local two-body operators that are not of zero-range character (e.g. gaussian, Coulomb, Yukawa, etc.). I: is also needed when calculating expectation values of any nonlocal (even one-body) operator. In paricular, to estimate the spurious rotational or translational energy content associated with 
a symmetry-breaking Slater determinant $|\Phi\rangle$ one evaluates $\left\langle\Phi\left|p^{2} / 2 m A\right| \Phi\right\rangle$ and $\left\langle\Phi\left|h^{2} J^{2} / 2 J\right| \Phi\right\rangle$, with usual notation, which implies the knowledge of the nonlocal part of $\rho$.

To describe low-energy dynamical nuclear processes in the adiabatic limit of the time-dependent Hartree-Fock (TDHF) approximation, one may cast [3] the equation of motion for any given collec. tive mode into the form of a HF problem with a constraining field. This field involves in general both $R$ and $\boldsymbol{p}$-dependent operators as e.g. in the well-known case of collective rotations [4]. In the realm of heavy-ion collisions now, the full phase-space one. body distribution $f(R, p)$ is required as a starting point for many theoretical descriptions of the reaction processes. Let us mention e.g. the evaluation of sub-threshold pion production [5], the fast proton emission mechanism [6], the modelisation of multifragmentation as a percolation phenomenon [7], or the stripping of a projectile nucleon in fragmentation reactions [8].

In these cases, as in many others, one strives for a simple yet accurate ansatz for the nonlocal part of the density matrix, thus avoiding a full microscopic evaluation which might exceed both the computational convenience and a detailed physical relevance. As existing approximations one must quote the infinite nuclear matter result (known as the Slater or Thomas-Fermi density matrix) and the so-called density matrix expansion (DME) either in the original formulation of Negele and Vautherin [9] or in the somewhat improved version of Campi and Bouyssy [10]. Whereas these approximations seem satisfactory for the description of static nuclear properties, they all lead to a Wigner transform which contains a step function discontinuity [11]. The latter may prove to be rather inconvenient, particularly for dynamical calculations. It is the aim of the present work to propose a simple parametrisation of the nonlocal behaviour of $\rho$ which does not suffer from the abovementioned deficiency.

As a first step one may approximate the dependence of $\rho$ on the relative coordinate $s$ in a gaussian form:

$\rho\left(r, r^{\prime}\right)=\rho(R, s)=\rho(R) \exp \left(-s^{2} / 2 \sigma^{2}\right)$.

Hereby $\rho(R)$ is the local density and the width $\sigma$ may still depend on the CM coordinate $\boldsymbol{R}$. Such an analyt- ical form is suggested by the general trend of exact density matrices obtained in HF calculations (see e.g. ref. [9] ). The simple form (1), however, turns out to be too crude. For instance, in the case of a harmonic oscillator potential the gaussian is multiplied by a polynomial in $s$. In the infinite nuclear-matter limit, on the other hand, the exact Slater density matrix has the form

$\rho\left(r, r^{\prime}\right)=\rho(s)=\rho_{0} 3 j_{1}\left(s k_{\mathrm{F}}\right) / s k_{\mathrm{F}}$,

$k_{\mathrm{F}}=\left(3 \pi^{2} \rho_{0}\right)^{1 / 3}$,

where $\rho_{0}$ is the nuclear matter density and $k_{\mathrm{F}}$ the Fermi momentum. Replacing $\rho_{0}$ by the local density $\rho(R)$ of a finite system leads to the so-called Slater approximation which is the starting point of the already mentioned DME approaches ${ }^{\ddagger 1}$.

As a slightly more sophisticated ansatz we thus propose the following modified gaussian density matrix (hereafter referred to as GDM)

$\rho(R, s)=\rho(R)\left(1-s^{2} / \alpha^{2}\right) \exp \left(-s^{2} / 2 \sigma^{2}\right)$.

Hereby the functions $\sigma(R)$ and $\alpha(R)$ are determined by the two following conditions:

(1) The local imposition of the correct kinetic energy density

$\tau^{*}(R)=\tau(R)-\frac{1}{4} \Delta \rho(R)=-\left[\Delta_{\mathrm{s}} \rho(R, s)\right]_{s=0}$,

where $\tau(R)$ is defined as in ref. [1]. This leads to

$1 / \sigma^{2}(R)=\tau^{*}(R) / 3 \rho(R)-2 / \alpha^{2}(R)$.

(2) The integrated projector identity of the density matrix

$\int \mathrm{d}^{3} R \int \mathrm{d}^{3} s|\rho(R, s)|^{2}=\int \mathrm{d}^{3} R \rho(R)$.

Together with the ansatz

$\alpha(R)=x / \hat{k}_{\mathrm{F}}(R)$,

with constant $x$ and $\hat{k}_{\mathrm{F}}(R)$ given by

$\hat{k}_{\mathrm{F}}(\boldsymbol{R})=\left[5 \tau^{*}(\boldsymbol{R}) / 3 \rho(\boldsymbol{R})\right]^{1 / 2}$,

one obtains a relatively simple algebraic equation for $x$ in terms of integrals involving $\rho(R)$ and $\hat{k}_{\mathrm{F}}(R)$ which we solve numerically [13]. This procedure

${ }^{\ddagger 1}$ A whole class of approximate forms for $\rho(R, s)$ which contains the DME approaches as well as eq. (1) has been proposed in ref. [12]. 
must of course be performed separately for neutrons and protons.

The density matrix (3) does not include any dependence on the angle $s \cdot \boldsymbol{R}$. (It could easily be built in at the cost of introducing more parameters.) We do not believe, however, that this is a serious drawback, since realistic HF density matrices depend rather weakly on this angle [9]. Note that in all DME approaches used so far $[9-12,14]$ the angle $s \cdot R$ is averaged out explicitly.

Our GDM ansatz could be very easily extended to reproduce the spin-orbit density $J(r)$ by simply adding to the polynomial term in eq. (3) an extra term is $\cdot(\sigma \times J) / 2 \rho(R)$, where $\sigma$ here is the Pauli spin matrix.

The local Fermi momentum $\hat{k}_{\mathrm{F}}(R)$, eq. (8), is the same as the one proposed by Campi and Bouyssy [10]. With eqs. (7), (8) the relation (5) takes the simple form

$1 / \sigma^{2}(R)=\left(\frac{1}{5}-2 / x^{2}\right) \hat{k}_{\mathrm{F}}^{2}(R)$.

The nonlocal part of the density matrix, eq. (3), thus only depends on the combination $s \hat{k}_{\mathrm{F}}(R)$. In the limit of semi-infinite nuclear matter, $\hat{k}_{\mathrm{f}}(R)$ reduces to $k_{\mathrm{F}}$ as given by eq. (2).

By the two above conditions our gaussian density matrix is completely determined through the knowledge of the local functions $\rho(R)$ and $\tau(R)$. These may in principle be taken from microscopic HF calculations as done in the DME approaches $[9,10]$.

In our present study we use a semiclassical method based on the so-called extended Thomas-Fermi (ETF) model [15] which allows to express $\tau(R)$ in terms of $\rho(R)$ and its gradients. The gaussian density matrix eq. (3) is thus determined by the local density $\rho(R)$ alone. The latter is obtained selfconsistently by performing density variational calculations [15] with a given effective interaction. The resulting density matrix $\rho(R, s)$ then represents a semiclassical approximation to the full microscopic density matrix obtained in a HF calculation with the same effective interaction. Note that our approach goes beyond the usual semiclassical $\hbar$-expansion of the Wigner function [16] in so far as it does not refer to a given one-body (HF) potential but directly to the local density and its derivatives. This allows in particular to perform selfconsistent density variational calculations.

It is well known that the ETF expansion of the functional $\tau[\rho]$ must be truncated at the order $\hbar^{4}$ for densities that fall off exponentially. Similarly the local momentum $\hat{k}_{\mathrm{F}}$, expressed in terms of $\tau^{*} / \rho$, can only be used in this context up to $\hbar^{2}$-terms. In practice one may froceed in the following way: first perform selfconsistent semiclassical calculations to determine the best possible local $\rho(R)$ (e.g. fourth-order ETF approach [15], as we have chosen to do here), then approximate the nonlocal part of $\rho$ through our GDM ansatz using $\hat{k}_{\mathrm{F}}$ determined by eq. (8) with $\tau[\rho]$ up to $\hbar^{2}$-terms. The function $\hat{k}_{\mathrm{F}}(R)$ then goes asymp. totically to a constant, if the density $\rho(R)$ falls off exponentially at large distances, whereas it tends to zero like $R^{-1 / \text { ? }}$ when using the exact quantummechanical densities $\rho$ and $\tau^{*}$ in eq. (8). Alternatively $\hat{k}_{\mathrm{F}}(R)$ can be determined by making use of the semiclassical densities obtained in the partial $\hbar$ resummation method [17]. In this case $\hat{k}_{\mathrm{F}}(\boldsymbol{R})$ is found to decrease, though faster than $R^{-1 / 2}$. We believe however, that the difficulties met in these semiclassical treatments of $\hat{k}_{\mathrm{F}}(R)$ are not significant in static or low-energy dyramical applications of our density matrix, as will also be demonstrated in the examples discussed belov'.

The projector identity $\rho^{2}=\rho$ of the density matrix is only imposecl in an integrated form by eq. (6). To investigate its local violation we have calculated the defect function

$$
\mathcal{D}(r)=\frac{1}{\rho_{0}}\left(\int \mathrm{d}^{3} r^{\prime}\left|\rho\left(r, r^{\prime}\right)\right|^{2}-\rho(r)\right) .
$$

The result is illistrated in fig. 1 for the two spherical nuclei ${ }^{40} \mathrm{Ca}$ and ${ }^{208} \mathrm{~Pb}$ which have been calculated using the $\mathrm{SkM}^{*}$ effective force [18]. For comparison we also show the defect function obtained with the Campi-Bouyssy DME ansatz [10] which also violates the projector identity.

We now turn to the behaviour of our GDM ansatz in momentum space. By a Fourier transformation of the density matrix (3) one gets readily the Wigner distribution function $\neq 2$

$$
\begin{aligned}
& f_{\mathrm{GDM}}(R, k)=(2 \pi)^{3 / 2} \rho \sigma^{3} \\
& \quad \times\left(1-3 \sigma^{2} / \alpha^{2}+k^{2} \sigma^{4} / \alpha^{2}\right) \exp \left(-\frac{1}{2} k^{2} \sigma^{2}\right),
\end{aligned}
$$

\footnotetext{
\$2 From now on we shall omit in our notation the explicit $\boldsymbol{R}$-dependence of the local functions $\rho, \alpha$ and $\sigma$.
} 


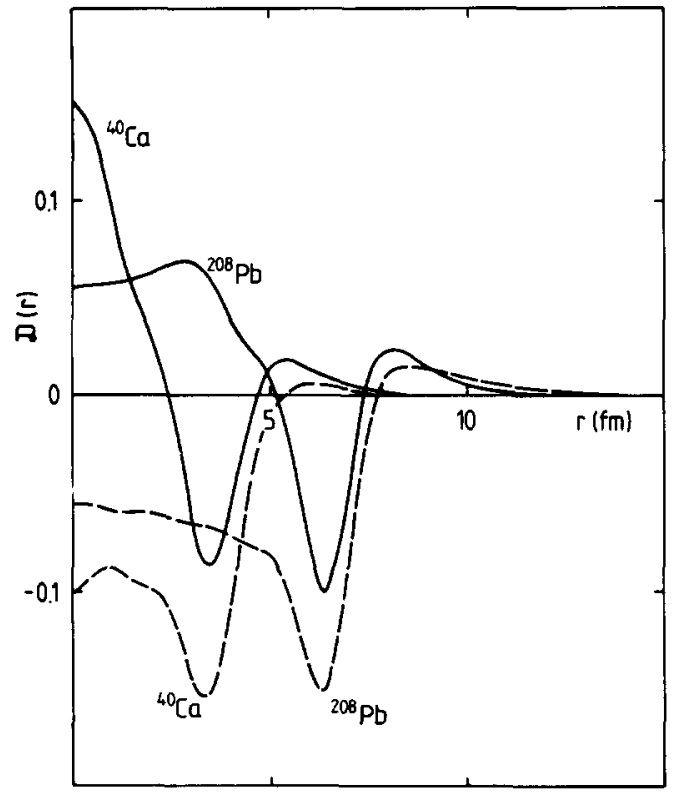

Fig. 1. Defect function $\mathcal{D}(r)$, eq. (8), for the GDM ansatz (solid) and the Campi-Bouyssy DME approach (dashed line). Curves shown for the proton distributions of ${ }^{40} \mathrm{Ca}$ and ${ }^{208} \mathrm{~Pb}$ (Skyrme SkM* force used).

with $\boldsymbol{k}=\boldsymbol{p} / \hbar$. Integrating $f$ with respect to $R$ yields the momentum distribution $\rho(k)$. Its zeroth and second moments are by construction the particle number and the kinetic energy, respectively.

One essential advantage of our ansatz (3) lies in the analytical form of its phase-space distribution (11). As already mentioned, the DME approaches [9, 10] lead to nonanalytical Wigner functions. The Campi-Bouyssy form leads in particular to

$f_{\mathrm{CB}}(R, k)=\left(6 \pi^{2} / \hat{k}_{\mathrm{F}}^{3}\right) \rho \theta\left(\hat{k}_{\mathrm{F}}-k\right)$,

with $\hat{k}_{\mathrm{F}}$ given by eq. (8). In a subsequent publication [14] the somewhat unphysical step function in eq. (12) has been replaced in an ad hoc way by a Fermi function whose width was adjusted to fit the fourth moment of $\rho(k)$. Unfortunately this entails a rather involved form for $\rho(R, s)$.

Various weighted moment distributions $k^{2} \rho(k)$ are displayed in fig. 2 for the nucleus ${ }^{208} \mathrm{~Pb}$ in the pure harmonic oscillator model case : together with the exact result [11] we show the distribution corresponding to the GDM and the Campi-Bouyssy DME

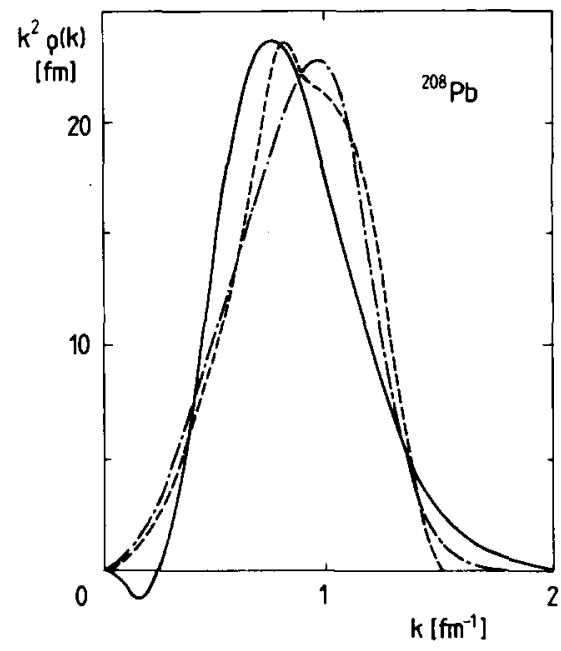

Fig. 2. Weighted momentum distribution $k^{2} \rho(k)$ of ${ }^{208} \mathrm{~Pb}$ within the pure harmonic oscillator model. Full line: GDM, dashed line: Campi-Bouyssy DME, dash-dotted line: exact result.

approach [10]. Although the latter yield exact zeroth and second moments of $\rho(k)$, they deviate from the exact curve. Part of this difference may be attributed to shell effects. The discontinuous step function character of the Campi-Bouyssy Wigner function results in a sharp cut-off behaviour of $\rho(k)$. The GDM ansatz, in turn, leads to an analytical distribution $\rho(k)$ with a somewhat too high tail. At small momenta, $\rho(k)$ is slightly negative which, of course, is unphysical. (This could in principle be avoided by choosing $x$ $\geqslant 5$.) This defect does, however, not seem to have any negative consequences for the applications considered below. Its importance in dynamical calculations remains to be checked.

As a first application we show in fig. 3 the Coulomb exchange energies obtained with our GDM approach for some spherical nuclei using the SkM* effective force. The exact results [19] have been rescaled assuming that the relative error due to the use of the Slater approximation ( $\sim \%$ in heavy nuclei) is independent of the force. We see that our GDM results are excellent, in fact far better than those obtained with both the Campi-Bouyssy [10] and the Slater approach.

The spurious center-of-mass energy inherent in the independent particle approximation is generally esti- 


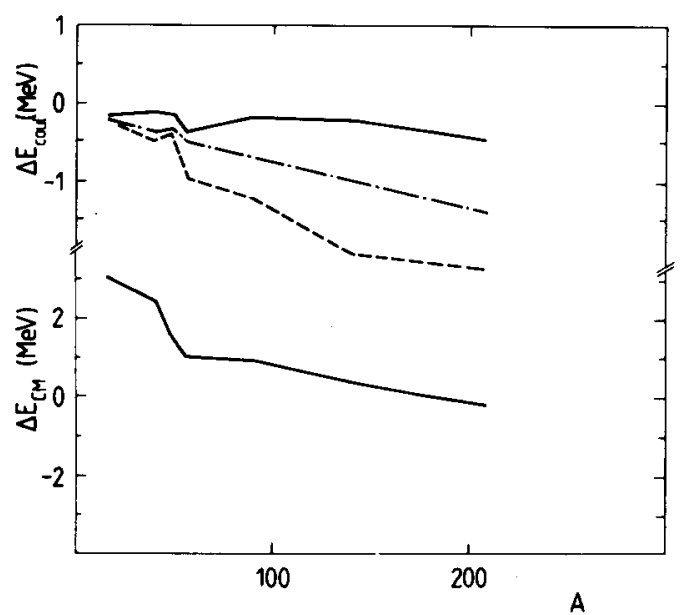

Fig. 3. Coulomb and center-of-mass exchange energies for various spherical nuclei versus $A$. Shown is the deviation between the approximate and the exact energies. Full line: GDM approach, dashed curve: Campi-Bouyssy DME, dash-dotted line: Slater approximation (Skyrme force SkM* used).

mated by

$E_{\mathrm{CM}}=\frac{1}{2 m A}\left\langle\Phi\left|\left(\sum_{i} p_{i}\right)^{2}\right| \Phi\right\rangle$,

and subtracted from the total HF energy. The operator $\left(\Sigma_{i} p_{i}\right)^{2}$ contains both a one-body $\left(\Sigma_{i} p_{i}^{2}\right)$ and a two-body part $\left(\Sigma_{i j} p_{i} p_{j}\right)$. The latter is omitted in most current HF calculations [20] for computational convenience, although this is known from a perturbative estimate [21] to lead to a drastic overestimation of $E_{\mathrm{CM}}$ in heavy nuclei. A selfconsistent study of this prescription remains, however, still to be done. In this context it is interesting to check how well our GDM ansatz is able to reproduce the exact CM energy correction. Indeed, the Slater as well as the DME approaches yield divergent results due to the step function discontinuity of their Wigner function, and therefore cannot be used in the calculation of $E_{\mathrm{CM}}$. Our GDM ansatz on the contrary, as seen in fig. 3 , yields a very nice reproduction of the expectation values obtained in the HF method. Our estimate for the full (i.e. direct plus exchange) CM energy yields in heavy nuclei a better reproduction of HF results than the quantal (harmonic oscillator) pocket formula of ref. [22]. The latter, however, leads to better results in light nuclei where our GDM ansatz slightly underestimates the full spurious energy as can be deduced from fig. 3.

As a final il.ustration we apply the GDM ansatz to the calculation of nuclear binding energies using the finite-range effective force D1 by Gogny [23]. Hereby one not only has to use the nonlocal density matrix in the calculation of the exchange energy, but the kinetic energy density functional $\tau[\rho]$ has to be determined in a consistent way [24]. Indeed, both the DME and our ISDM approach lead to a variable effective nucleon mass which enters explicitly the ETF gradient expansion [14] of $\tau[\rho]$.

First of all we have computed the exchange potential energy assciciated with the D1 force in infinite nu. clear matter for $k_{\mathrm{F}}=1.36 \mathrm{fm}^{-1}$. The resulting value $(-31.60 \mathrm{MeV})$ compares rather well with the exact one $(-31.37 \mathrm{NeV})$. Releasing the integrated $\rho^{2}=\rho$ condition (eq. (6)), but choosing $x$ in eq. (7) to reproduce the exact exchange energy density up to order $\left(\mu k_{\mathrm{F}}\right)^{4 \neq 3}$, on : obtains $x=(350)^{1 / 4}=4.33$ (instead of 4.78) and th erewith an exchange energy which reproduces the exact one within less than $10 \mathrm{keV}$. This prescription (with $x=4.33$ ) thus will lead to the correct volume energy coefficient $a_{\mathrm{v}}=-16.3 \mathrm{MeV}$ in the binding energy of finite nuclei.

Turning now to finite nuclei, we have evaluated the selfconsist $\mathrm{nt}$ semiclassical local density $\rho$ within the ETF framework, adapted to the Gogny D1 force [24]. Assumin; GDM density matrices we have computed the total binding energies of some spherical nuclei listed in talsle 1 . The spurious CM energies have been determined as above and subtracted from the calculated energies. Obviously the total semiclassical energies have to be corrected for shell effects which we have taken from the selfconsistent calculations of ref. [17] using the Skyrme SkM* force. As compared to the results of Hartree-Fock-Bogolyubov ${ }^{\neq 4}$ calculations [23], the results corresponding to the $\rho^{2}$ $=\rho$ condition laad to an overbinding of $1-20 \mathrm{MeV}$ from light to haavy nuclei. On the other hand, the calculations with $x=4.33$ yield a total energy which is in far better agreement with the HF energies. The

\footnotetext{
$\neq 3$ Where $\mu$ is the range of the gaussian force $\sim \exp \left(-s^{2} / \mu^{2}\right)$.

$\neq 4$ Note that pairing correlations are not expected to play any significant role in such spherical nuclei.
} 
Table 1

Total semiclassical binding energies obtained for various spherical nuclei with Gogny's gaussian force D1. First line: Campi-Bouyssy DME approach, second line: GDM ansatz with $\rho^{2}=\rho$, third line: GDM ansatz with $x=4.33$ (see text), fourth line: HF energies of ref. [23].

\begin{tabular}{|c|c|c|c|c|c|}
\hline \multirow[t]{2}{*}{ Method } & \multicolumn{5}{|l|}{ Nuclei } \\
\hline & ${ }^{16} \mathrm{O}$ & ${ }^{40} \mathrm{Ca}$ & ${ }^{48} \mathrm{Ca}$ & ${ }^{90} \mathrm{Zr}$ & ${ }^{208} \mathrm{~Pb}$ \\
\hline Campi-Bouyssy & -118.2 & -326.4 & -397.8 & -777.6 & -1648.7 \\
\hline $\begin{array}{l}\text { GDM } \\
\qquad \rho^{2}=\rho\end{array}$ & -128.3 & -337.9 & -412.3 & -785.3 & -1653.6 \\
\hline $\begin{array}{l}\text { GDM } \\
\qquad x=4.33\end{array}$ & -129.3 & -334.3 & -407.2 & -773.8 & -1623.6 \\
\hline $\mathrm{HF}$ & -127 & -338 & -411 & -779 & -1633 \\
\hline
\end{tabular}

slight underbinding observed here is only a surface effect and, indeed, roughly proportional to $A^{2 / 3}$. In this table we have also reported the energies obtained in the Campi-Bouyssy approach which overbind heavy and significantly underbinds light nuclei. Since we know the HF results only approximately (they are taken from a figure in ref. [23]) and since the shell correction energies have been taken from a different effective force, we expect an uncertainty in the differences to the HF energies of $\pm(1-2) \mathrm{MeV}$.

It may be concluded that our GDM ansatz yields a reproduction of various nuclear properties which is of an equivalent if not better quality as compared to Slater or DME approaches. Furthermore being free from the unphysical step function character of the Wigner function present in the latter, it allows a correct evaluation of quantities otherwise not accessible such as the exchange center-of-mass spurious energy. Obviously, the full relevance of the proposed phasespace distribution remains to be assessed in dynamical calculations which are currently undertaken. The value of the whole approach does, by construction, not extend beyond the validity of any HF approximation whose ability to reproduce both local and nonlocal experimental one-body density matrices has recently been questioned [25].

The authors are most grateful to C. Guet for useful discussions. This work has resulted from numerous visits of the authors to each others' laboratories and GANIL. We would like to express warmly our gratitude to B. Bonnier, C. Detraz, E. Elbaz and W. Weise for the fruitful hospitality extended to us in each of their laboratories.

\section{References}

[1] D. Vautherin and D.M. Brink, Phys. Rev. C5 (1972) 626.

[2] Y.M. Engel, D.M. Brink, K. Goeke, S.J. Krieger and D. Vautherin, Nucl. Phys. A249 (1975) 215.

[3] M.J. Giannoni and P. Quentin, Phys. Rev. C21 (1980) 2060.

[4] D.J. Thouless and J.G. Valatin, Nucl. Phys. 31 (1962) 211.

[5] C. Guet and M. Prakash, Nucl. Phys. A428 (1984) 119.

[6] C. Gregoire, B. Remaud, F. Scheuter and F. Sebille, Nucl. Phys. A436 (1985) 365.

[7] X. Campi and J. Desbois, Contrib. XXIII Intern. Winter Meeting on Nuclear physics (Bormio, 1985), Orsay preprint, IPNO/TH 85-9.

[8] J. Hüfner and M.C. Nemes, Phys. Rev. C23 (1981) 2538.

[9] J.W. Negele and D. Vautherin, Phys. Rev. C5 (1972) 1472; C11 (1975) 1031.

[10] X. Campi and A. Bouyssy, Phys. Lett. B 73 (1978) 263.

[11] See e.g. J. Martorell and E. Moya de Guerra, Ann. Phys. 158 (1984) 1 , and references therein.

[12] R.K. Bhaduri and L. Zaifman, Can. J. Phys. 76 (1979) 1990.

[13] J. Meyer, J. Bartel, M. Brack and P. Quentin, Proc. Topical Meeting on Phase space approach to nuclear dynamics (Triest, 1985) (World Scientific, Singapore), to be published.

[14] X. Campi and A. Bouyssy, Nucleonika 24 (1979) 1.

[15] See e.g. M. Brack, C. Guet and H.-B. Håkansson, Phys. Rep. 123 (1985) 275, and references therein.

[16] N.L. Balazs and G.G. Zipfel, Ann. Phys. (NY) 77 (1973) 139;

B.K. Jennings, Phys. Lett. B 74 (1978) 139; 
B. Grammaticos and A. Voros, Ann. Phys. (NY) 123 (1979) 359;

M. Durand, V.S. Ramamurthy and P. Schuck, Phys. Lett. B 113 (1982) 116;

M. Prakash, S. Shlomo and V.M. Kolomietz, Nucl. Phys. A370 (1981) 30.

[17] J. Bartel and M. Vallieres, Phys. Lett. B 114 (1982) 30; J. Bartel, Ph.D. Thesis, University of Regensburg (1984); and to be published.

[18] J. Bartel, P. Quentin, M. Brack, C. Guet and H.-B. Håkansson, Nucl. Phys. A386 (1982) 79.

[19] C. Titin-Schneider and P. Quentin, Phys. Lett. B 49 (1974) 397.
[20] See e.g. P. Quentin and H. Flocard, Annu. Rev. Nucl. Part. Sci. 28 (1978) 523.

[21] See e.g. C M. Ko, H.C. Pauli, M. Brack and G.E. Brown, Nucl. Phy s. A236 (1974) 269.

[22] M.N. Butler, D.W.L. Sprung and J. Martorell, Nucl. Phys. A422 (19:34) 157.

[23] D. Gogny in: Nuclear selfconsistent fields, eds. G. Ripka and M. Porneuf (North-Holland, Amsterdam, 1975) p. $\lesssim 33$;

J. Decharge and D. Gogny, Phys. Rev. C21 (1980) 1568.

[24] S. Aicher and M. Brack, to be published.

[25] M. Jaminon, C. Mahaux and H. Ngo, Phys. Lett. B 158 (1985) 103. 